\title{
Multifaceted COVID-19-Related Stressors and Associations with Indices of Mental Health, Well-being, and Substance Use Among Young Adults
}

\author{
Scott Graupensperger ${ }^{1}$ (D) . Jennifer M. Cadigan ${ }^{1} \cdot$ Clare Einberger $^{2} \cdot$ Christine M. Lee $^{1}$
}

Accepted: 1 July 2021 /Published online: 4 August 2021

(C) The Author(s), under exclusive licence to Springer Science+Business Media, LLC, part of Springer Nature 2021

\begin{abstract}
Alongside direct health concerns pertaining to the SARS-CoV-2 virus, the stressors and life disruptions associated with the COVID-19 pandemic may provoke secondary concerns for health and well-being. The implications of COVID-19-related stressors may be particularly salient for young adults, who are at higher-risk for mental health concerns and substance use behaviors. We developed a multifaceted scale that assessed distinct domains of COVID-19-related stressors and examined associations between these stressors and indices of mental health, well-being, and substance use (alcohol and marijuana use). In AprilJune of 2020, 1181 young adults were recruited from two- and four-year colleges to participate in this study $\left(M_{\text {age }}=20.40 ; 59.95 \%\right.$ women $)$. Exploratory factor analysis identified five domains of COVID-19-related stressors: job insecurity, social/relational, financial, illnessrelated, and school-related. The overall pattern of results indicated that COVID-19-related stressors were related to poorer mental health and well-being. Social/relational stressors emerged as a primary concern most strongly associated with indices of mental health and alcohol use, relative to other stressors. However, illness-related stressors and school-related stressors were associated with less alcohol use. Stressors associated with the COVID19 pandemic may impact young adults' health and well-being but disentangling various stressor domains informs more tailored intervention and prevention strategies.
\end{abstract}

Keywords SARS-CoV-2 $\cdot$ Alcohol $\cdot$ Marijuana $\cdot$ Depression $\cdot$ Anxiety $\cdot$ Sleep $\cdot$ College students

The COVID-19 pandemic has been a highly distressing time and has had detrimental impacts on multiple domains of daily life. Young adults — particularly college students — may be especially susceptible to negative pandemic-related experiences (i.e., beyond the burden directly associated with the SARS-CoV-2 virus). Disruptions associated with

Christine M. Lee

leecm@uw.edu

1 Department of Psychiatry and Behavioral Sciences, University of Washington, 1100 NE 45th St., Suite 300, Seattle, WA 98105, USA

2 School of Medicine, University of Washington, University of Washington, 1959 NE Pacific St, Seattle, WA 98195, USA 
campus closures and loss of employment have been salient among young adults as this age group has been disproportionately affected. For example, unemployment for young adults rose from $8.0 \%$ in February 2020 to $25.3 \%$ in May 2020 (Kochnar, 2020). Survey data has also shown that young adults are more concerned than older adults about paying for basic needs, affording housing, and paying for medical bills during the pandemic. As such, it is critical to examine how stressors related to the COVID-19 pandemic have impacted young adults' mental health and well-being.

Young adults are at high-risk for mental health problems, sleep problems, and substance use (Cadigan, et al., 2019a, 2019b; Kessler et al., 2005; Weitzman, 2004; Wu et al., 2007). Stress is one of the most frequently reported hindrances to young adult well-being, and is a major contributing factor to these concerns (Bovier et al., 2004; Tworoger et al., 2005). More than half (57\%) of college students report 'more than average' or 'tremendous' levels of stress in the past year (ACHA, 2018). Nearly one-third (32\%) of young adults report that stress negatively impacts their academic performance, followed by anxiety (26\%) and sleep difficulties (20\%) (ACHA, 2018). Stress has also been shown to be associated with negative mental health outcomes, including both depression and anxiety (Serido et al., 2004).

The stay-at-home restrictions and social distancing policies put in place to reduce the spread of COVID-19 may have negative effects on mental health (Holmes et al., 2020; Killgore et al., 2020; Pfefferbaum \& North, 2020). Relative to other age groups, researchers have found that the COVID-19 pandemic may be having especially salient negative effects on young adults, as reports show that this age group has experienced the greatest increase in psychological distress (McGinty et al., 2020). The rates of loneliness among young adults have also increased more than other adult age groups (Luchetti et al., 2020), especially for young adults with greater COVID-19-related social or relational concerns and those who had more social connectedness prior to the pandemic (Lee et al., 2020). Indeed, the limited social contact has been a major challenge for young adults with recent findings showing that college students who reported less social support and connectedness with their peer groups during the pandemic reported lower well-being and more symptoms of depression (Graupensperger et al., 2020). Beyond the social aspects, concerns regarding illness from the disease itself can have negative impacts on mental health. For example, a study conducted in China found that college students with greater perceived risk of infection reported more symptoms of depression (Li et al., 2020).

Alongside mental health, another secondary health concern during the COVID-19 pandemic has been potential increases in substance use (Clay \& Parker, 2020). Alcohol sales have been high during the pandemic, as one study found alcohol sales have increased by $55 \%$ in March 2020, compared to that same time in 2019 (Bremner, 2020). Whereas recent cross-sectional evidence indicated that most college students self-reported drinking the same or less than before the pandemic (Graupensperger et al., 2021a, b), a longitudinal study that examined intraindividual changes in a community sample of young adults' alcohol use from January 2020 to April/May 2020 revealed that the patterns of use have shifted such that many are drinking more frequently but consuming less drinks per occasion (Graupensperger et al., 2021a). Moreover, researchers found that young adults' motives for substance use have also shifted during the pandemic. Pertaining to alcohol use, depression coping motives have increased while drinking for social, enhancement, and conformity reasons have decreased and, pertaining to marijuana use, using for boredom reasons increased while celebration motives decreased (Graupensperger et al., 2021a). Young adults may be more motivated to drink during the pandemic to self-medicate and cope with distress and uncertainty (Khantzian, 1997; Lechner et al., 2020). 
A recent study examined psychological distress related to COVID-19 for adults (e.g., COVID-19 negatively impacting psychological health; feeling depressed due to COVID19) (Rodriguez et al., 2020). Findings suggested that greater COVID-19-related psychological distress was associated with increased drinking frequency and heavy drinking episodes for both men and women. However, there were some gender-differences, such that distress was associated with greater peak number of drinks and typical number of drinks consumed for only women. This suggests that while both men and women were drinking more frequently in response to COVID-19-related distress, women were drinking more on typical and heavy drinking occasions in response to the distress. As the average age of participants in this study by Rodriguez and colleagues was 41.7 years $(\mathrm{SD}=10.39)$, less is known regarding how COVID-19 stressors are associated with substance use behaviors of young adults.

\section{Present Study}

The full scope of how COVID-19 has impacted health and well-being may not be known for years to come. Beyond the actual disease, the public health containment and mitigation strategies have had adverse effects on numerous domains of life for many people, including work, education, social relationships, and finances, to name a few. The present study examined the stressors young adult college students have experienced related to the COVID-19 pandemic and how these concerns are associated with indices of mental health and wellbeing. We developed a measure to assess different domains of COVID-19-related stressors and examined how these stressors may be associated with mental health, physical health, sleep, and substance use (i.e., alcohol and marijuana use) among two- and four-year college students. We generally anticipated that increased endorsement of COVID-19-related stressors would be associated with poorer indices of mental health and well-being and greater use of alcohol and marijuana (Lee et al., 2020; Rodriguez et al., 2020). However, because the pandemic has potentially impacted multiple domains of young adults' lives, we focused on exploring which domains are of most concern to students in terms of negative impact on health and well-being. This timely information provides guidance for interventions and other translational public health strategies.

\section{Method}

\section{Procedures and Participants}

Data for the present study were collected within the initial screening survey for an intensive longitudinal study that is testing a mobile phone intervention for reducing high-risk college student drinking (Clinical Trial \#NCT04213846). As part of the parent study, 5549 students were randomly selected from the registrar's list from three colleges in the Northwest USA (i.e., one large four-year public university and two community colleges) and sent e-mail invitations to complete the screening survey which, in addition to the necessary screener items, also included measures/items that are relevant to the present study. Invitation e-mails were sent out between April 6 and May 15 of 2020, and the survey closed on June 11 of 2020. In the state that data were collected, a stay-at-home order went into effect March 23, 2020 at which time all classes moved to an online format for students attending 
Table 1 Exploratory factor analysis of COVID-19 stressor items $(N=1181)$

\begin{tabular}{|c|c|c|c|c|c|c|c|}
\hline \multirow{2}{*}{ Prompt: How concerned are you about the novel Coronavirus (COVID-19)... } & \multicolumn{5}{|c|}{ Factor } & \multirow{2}{*}{$\begin{array}{l}\text { Item } \\
\text { Mean }\end{array}$} & \multirow{2}{*}{$\begin{array}{c}\text { Item } \\
\text { SD }\end{array}$} \\
\hline & 1 & 2 & 3 & 4 & 5 & & \\
\hline \multicolumn{8}{|l|}{ 1. Job Insecurity Stressors $(\alpha=.89)$} \\
\hline Causing you to lose your job, e.g. Employer laying you off? & 0.91 & -0.01 & -0.02 & 0.03 & 0.00 & 2.33 & 1.51 \\
\hline Causing a decrease in hours for your job? & 0.92 & 0.04 & -0.02 & -0.02 & 0.00 & 2.36 & 1.57 \\
\hline Causing you to lose your job-related benefits, e.g. Health insurance, paid sick leave? & 0.59 & -0.09 & 0.27 & 0.03 & 0.07 & 1.85 & 1.34 \\
\hline \multicolumn{8}{|l|}{ 2. Social/Relational Stressors $(\alpha=.76)$} \\
\hline Making you feel isolated or alone? & -0.01 & 0.79 & 0.03 & 0.04 & -0.02 & 3.07 & 1.36 \\
\hline Placing a strain on your social life? & 0.02 & 0.74 & 0.02 & -0.04 & 0.02 & 3.21 & 1.35 \\
\hline Placing a strain on relationships with those you live with? & 0.04 & 0.44 & 0.08 & 0.04 & 0.22 & 2.56 & 1.37 \\
\hline \multicolumn{8}{|l|}{ 3. Financial Stressors $(\alpha=.83)$} \\
\hline Affecting your ability to pay rent? & 0.00 & 0.02 & 0.88 & -0.03 & 0.01 & 2.09 & 1.45 \\
\hline Causing you to not have enough food to eat? & -0.02 & -0.02 & 0.74 & 0.10 & 0.08 & 1.94 & 1.24 \\
\hline Negatively affecting your financial situation? & 0.32 & 0.17 & 0.48 & 0.01 & -0.06 & 3.04 & 1.44 \\
\hline \multicolumn{8}{|l|}{ 4. Illness-related Stressors $(\alpha=.76)$} \\
\hline Making you sick? & 0.03 & -0.08 & 0.00 & 0.90 & 0.01 & 2.48 & 1.13 \\
\hline Making your friends or family sick? & -0.05 & 0.15 & 0.05 & 0.63 & -0.06 & 3.59 & 1.17 \\
\hline Making you afraid to leave your house? & -0.02 & 0.25 & 0.04 & 0.51 & 0.03 & 2.60 & 1.23 \\
\hline \multicolumn{8}{|l|}{ 5. School-related Stressors $(\alpha=.74)$} \\
\hline Affecting your ability to stay in school? & 0.01 & -0.02 & 0.08 & -0.01 & 0.75 & 2.40 & 1.46 \\
\hline Causing your college classes to move to online instruction? & 0.01 & 0.30 & -0.12 & -0.05 & 0.42 & 3.78 & 1.39 \\
\hline \multicolumn{8}{|l|}{ Did Not Load Onto any Factor (Loadings $<.40$ ) } \\
\hline making you feel overwhelmed with news and information? & 0.11 & 0.38 & -0.08 & 0.24 & 0.25 & 2.86 & 1.32 \\
\hline making you feel overwhelmed at work? & 0.11 & 0.18 & 0.00 & 0.14 & 0.28 & 2.14 & 1.39 \\
\hline
\end{tabular}

Note: Item responses ranged from 1 (Not at all) to 5 (Extremely). Factor loadings estimated using Oblimin rotation. The five-factor model achieved a simple structure with no cross-loadings. Root mean square of residuals $=.03$, root mean squared error of approximation $=.05$, and Tucker-Lewis Index $=.95$. Loadings larger than .40 are bold

these three colleges. Completion of this survey was remunerated with a $\$ 15$ e-gift card. IRB approval was obtained, and no adverse events were reported.

Responses were received from 1181 college students (21.28\% response rate). Participants ranged in age from 18 to $25(M=20.40, S D=1.78), 59.95 \%$ reported being female, and the ethnoracial composition of the sample is as follows: $50.55 \%$ White, $25.74 \%$ Asian/Asian-American, $22.78 \%$ other non-Hispanic, and $0.93 \%$ Hispanic. Participants were enrolled in both four-year (58.09\%) and two-year (41.91\%) higher education institutions. Pertaining to current employment status, using a check-all-that-apply format, $34.14 \%$ reported working part-time, $8.00 \%$ full-time, $4.56 \%$ working more than one job, $6.53 \%$ working as an intern/apprentice, $12.90 \%$ unemployed and actively seeking work, and $10.23 \%$ unemployed and not seeking work. ${ }^{1}$

\section{Measures}

COVID-19-Related Stressors Sixteen items were created by a team of experts in young adult development to be used in the present study. Items were selected based on domains related to potential impact on young adults' lives, based on the most pressing concerns for young adults and college students during the early-stages of the COVID-19 pandemic. The stem for these questions included: "The questions in this scale ask you about your feelings and thoughts at this time during the COVID-19 pandemic. How concerned are you about the novel coronavirus (COVID-19)..." Response ranges from $1=$ Not at all to $5=$ Extremely. These items are listed in Table 1 and additional details regarding this instrument and individual items is provided in the online supplemental materials.

\footnotetext{
1 Other employment response options were available (e.g., homemaker, receiving disability income) but were endorsed by very few participants $(\sim 1 \%)$ and are thus not reported.
} 
Depression and Anxiety Symptoms The four-item Patient Health Questionnaire-4 was used to assess past month depression and anxiety symptoms (Kroenke et al., 2009). Two items assessed symptoms of depression ("Little interest or pleasure in doing things"; "Feeling down, depressed, or hopeless") and two items assessed symptoms of anxiety ("Feeling nervous, anxious, or on edge", "Not being able to stop or control worrying"). Response options ranged from $0=$ Not at all to $3=$ Nearly every day, and items were summed within each subscale. Spearman-Brown reliability estimates were $\alpha=0.86$ for depression and $\alpha=0.91$ for anxiety.

Perceived Mental Health, Physical Health, and Sleep Quality Single-item scales were adapted from previous research on college and community college students (Cadigan et al., 2020) to assess subjective perceptions of well-being pertaining to mental health, physical health, and sleep quality in the last month. On a scale ranging from $1=$ Excellent to $5=$ Poor, participants responded to the following questions: (a) "In general, how would you say your mental health was in the past month?", (b) "In general, how would you say your physical health was in the past month?", and (c) "In general, how would you say your sleep quality was in the past month?" Higher scores reflected poorer perceptions of health and well-being in these respective domains.

Alcohol and Marijuana Use Two indices of alcohol use were assessed: typical number of drinks per week and number of heavy episodic drinking occasions (i.e., $4+$ drinks in a row for women $/ 5+$ drinks in a row for men). Typical number of drinks was assessed using the Daily Drinking Questionnaire (Collins et al., 1985), which asks participants to report the number of drinks they typically consumed on each day of the week within the past month, and all drinks are summed to create an index of weekly drinking. Number of heavy episodic drinking occasions was assessed using a single item adapted from the Monitoring the Future questionnaire (Bachman et al., 2012), in which participants reported the number of occasions that they consumed $4+/ 5+$ drinks in a row, for women and men, respectively. Marijuana use frequency was assessed using a single item that asked: "On how many occasions, if any, have you used marijuana (weed, pot) or hashish (hash, hash oil) during the last 30 days?" Response options ranged from $0=$ Zero occasions to $6=40$ or more occasions (Bachman et al., 2012).

\section{Analyses}

\section{Exploratory Factor Analysis (EFA)}

In an initial step, EFA was used to identify the latent relational structure among the sixteen COVID-19-related stressor items (i.e., to identify factors or subdomains of COVID19-related stressors). Parallel analysis was used to identify the number of unique factors, which produced a scree plot that is available in the online supplement. EFA was specified to have oblique/oblimin rotation given our expectation that the identified factors would be correlated, and ordinary least squared factoring was selected as this approach derives solutions through iterative eigendecomposition. Cutoffs for factor loadings were predetermined to be at 0.40 . EFA was conducted in R using the 'GPArotation' package (Bernaards \& Jennrich, 2005). 


\section{Mental Health and Well-Being}

Multiple linear regression was used to estimate associations between COVID-19-related stressors and indices of mental health and well-being. This entailed five separate models to examine each outcome of interest. In all models, we statistically controlled for participants' age, birth sex, and four- or two-year college status.

\section{Alcohol and Marijuana Use}

The three outcome variables pertaining to substance use - typical number of drinks, heavy episodic drinking occasions, and marijuana use frequency — are positively skewed non-negative integers that require the use of a nuanced count regression approach (i.e., Poisson regression). Moreover, Vuong model-fitting tests (Vuong, 1989) revealed that all three of these outcome variables were zero-inflated with conditional variance exceeding the conditional means (i.e., overdispersion). As such, zero-inflated Poisson (ZIP) regression was used to estimate the associations between COVID-19-related stressors and substance use behaviors.

ZIP models are a specific type of mixture modeling that entails two separate analytic steps. First, these models entail a logistic portion that estimates the likelihood of the observation or response being a value of zero as opposed to any other positive value. In other words, this step estimates the extent that covariates predict a zero-value response (e.g., an alcohol abstainer). After accounting for excess zeros in the logistic portion, the count portion of model estimates the association between the covariates and all non-negative responses on the outcome. We statistically controlled for participants' age, birth sex, and four-year/two-year college status in these models. Although different covariates can be specified for the logistic and count portions, we included the same covariates for both dimensions as there was no theoretical rationale for specifying covariates at just one level.

\section{Results}

\section{EFA on COVID-19-Related Stressor Items}

Parallel analysis and visual inspection of the scree plot (available in the online supplement) indicated that a five-factor model was the best to fit to these data. Indeed, a simple structure was identified in which no items cross-loaded onto more than one factor (Table 1). We interpreted these five factors as stressors related to: job insecurity, social/relational, financial, illness-related, and school-related. Two items did not load onto any of the five factors and were therefore not included in subsequent models (see Table 1).

\section{Descriptive Statistics}

Descriptive statistics and bivariate correlations are displayed in Table 2. On average, participants reported relatively few symptoms of depression and anxiety with mean scores falling below the recommended cut-off for further evaluation (i.e., scores of 3 or higher; Kroenke et al., 2009). However, 28.21\% of the sample scored above this cutoff for depressive symptoms and $35.26 \%$ scored above this cutoff for anxiety symptoms, which are percentages similar to those found in other studies (i.e., near $30 \%$; 
Table 2 Bivariate correlations and descriptive statistics $(N=1181)$

\begin{tabular}{|c|c|c|c|c|c|c|c|c|c|c|c|c|c|c|c|}
\hline & 1 & 2 & 3 & 4 & 5 & 6 & 7 & 8 & 9 & 10 & 11 & 12 & 13 & 14 & 15 \\
\hline \multicolumn{16}{|l|}{ 1. Depression Symptoms } \\
\hline 2. Anxiety Symptoms & $.62 * *$ & & & & & & & & & & & & & & \\
\hline 3. Poor Subjective Mental Health & $.65^{* *}$ & $.59^{* *}$ & & & & & & & & & & & & & \\
\hline 4. Poor Subjective Physical Health & $.33 * *$ & $.26 * *$ & $.43 * *$ & & & & & & & & & & & & \\
\hline 5. Poor Sleep Quality & $.41 * *$ & $.38 * *$ & $.52 * *$ & $.36 * *$ & & & & & & & & & & & \\
\hline 6. Typical Drinks per Week & $.07^{*}$ & $.07^{*}$ & $.07 *$ & .01 & .02 & & & & & & & & & & \\
\hline 7. HED Occasions & $.09 * *$ & $.10^{* *}$ & $.11 * *$ & .04 & $.07 *$ & $.75^{* *}$ & & & & & & & & & \\
\hline 8. Marijuana Use Frequency & $.21 * *$ & $.19 * *$ & $.19 * *$ & $.06^{*}$ & $.09 * *$ & $.35^{* *}$ & $.32 * *$ & & & & & & & & \\
\hline 9. Job Insecurity Stressors & $.14 * *$ & $.16^{* *}$ & $.09^{* *}$ & .02 & $.07^{*}$ & $.09^{* *}$ & $.08^{* *}$ & $.07 *$ & & & & & & & \\
\hline 10. Social/Relational Stressors & $.35^{* *}$ & $.34 * *$ & $.35 * *$ & $.13 * *$ & $.20^{* *}$ & $.16^{* *}$ & $.12 * *$ & $.09 * *$ & $.27 * *$ & & & & & & \\
\hline 11. Financial Stressors & $.23 * *$ & $.23 * *$ & $.19 * *$ & $.09^{* *}$ & $.13 * *$ & $.07^{*}$ & .05 & $.11 * *$ & $.63 * *$ & $.34 * *$ & & & & & \\
\hline 12. Illness-related Stressors & $.14 * *$ & $.21 * *$ & $.14 * *$ & $.13 * *$ & $.09 * *$ & .02 & -.01 & .04 & $.28^{* *}$ & $.39 * *$ & $.37 * *$ & & & & \\
\hline 13. School-related Stressors & $.27 * *$ & $.24 * *$ & $.23 * *$ & $.11 * *$ & $.20 * *$ & .02 & -.02 & .05 & $.23 * *$ & $.46 * *$ & $.30^{* *}$ & $.22 * *$ & & & \\
\hline 14. Age & $.06^{*}$ & $.08^{* *}$ & $.07^{*}$ & $.08^{* *}$ & .03 & $.21 * *$ & $.16^{* *}$ & $.11^{* *}$ & $.11^{* *}$ & -.04 & $.13^{* * *}$ & $.07^{*}$ & $-.09 * *$ & & \\
\hline 15. Student $(2$-year $=0$, 4-year $=1)$ & $-.08^{* *}$ & $-.06 *$ & $-.08 * *$ & $-.11^{* *}$ & $-.14 * *$ & $.14^{* *}$ & $.08 * *$ & $-.10^{* *}$ & $-.11 * *$ & .05 & $-.13^{* *}$ & -.03 & -.03 & $-.14^{* *}$ & \\
\hline 16. Birth $\operatorname{Sex}(M=0, F=1)$ & $.09^{* *}$ & $.20^{* *}$ & $.19^{* *}$ & $.11^{* *}$ & $.06^{*}$ & $-.06^{*}$ & -.01 & $-.08^{* *}$ & $.10^{* *}$ & $.16^{* *}$ & $.07 *$ & $.15^{* *}$ & $.09^{* *}$ & .01 & -.03 \\
\hline Mean & 1.94 & 2.20 & 3.11 & 2.44 & 3.28 & 2.93 & 0.50 & 1.12 & 2.18 & 2.94 & 2.35 & 2.89 & 3.09 & 20.4 & 0.58 \\
\hline SD & 1.53 & 1.75 & 1.17 & 1.09 & 1.17 & 5.23 & 1.35 & 1.90 & 1.33 & 1.11 & 1.19 & 0.97 & 1.20 & 1.78 & 0.49 \\
\hline Range & $0-6$ & $0-6$ & $1-5$ & $1-5$ & $1-5$ & $0-25$ & $0-12$ & $0-6$ & $1-5$ & $1-5$ & $1-5$ & $1-5$ & $1-5$ & $18-25$ & -- \\
\hline
\end{tabular}

Note: HED, Heavy episodic drinking. 1. Typical number of weekly drinks was Winsorized at 3 SD above the mean to reduce the influence of extreme values (Tabachnik \& Fidell, 2019). ${ }^{*} p<.05 . * * p<.01$

Table 3 Regression models estimating associations between COVID-19 stressors and indices of health and well-being $(N=1181)$

\begin{tabular}{|c|c|c|c|c|c|}
\hline Independent Variables & $\begin{array}{l}\text { Depression } \\
\text { Symptoms }\end{array}$ & $\begin{array}{c}\text { Anxiety } \\
\text { Symptoms }\end{array}$ & $\begin{array}{c}\text { Poor } \\
\text { Subjective } \\
\text { Mental } \\
\text { Health } \\
\end{array}$ & $\begin{array}{c}\text { Poor } \\
\text { Subjective } \\
\text { Physical } \\
\text { Health }\end{array}$ & $\begin{array}{c}\text { Poor Sleep } \\
\text { Quality }\end{array}$ \\
\hline & $\beta(S E)$ & $\beta(S E)$ & $\beta(S E)$ & $\beta(S E)$ & $\beta(S E)$ \\
\hline Age & $.06(.02)^{*}$ & $.07(.03)^{*}$ & $.05(.02)^{* *}$ & $.04(.02)^{*}$ & $.01(.02)$ \\
\hline Birth $\operatorname{Sex}(M=0, F=1)$ & $.09(.09)$ & $.50(.10)^{* * *}$ & $.36(.07)^{* * *}$ & $.19(.07)^{* *}$ & $.07(.07)$ \\
\hline Student Status $(2$-year $=0,4$-year $=1)$ & $-.23(.09)^{* *}$ & $-.15(.10)$ & $-.17(.07)^{* *}$ & $-.23(.07)^{* * *}$ & $-.33(.07)^{* * *}$ \\
\hline Job Insecurity Stressors & $-.06(.04)$ & $-.04(.05)$ & $.08(.03)^{* *}$ & $.10(.03)^{* *}$ & $.06(.03)$ \\
\hline Social/Relational Stressors & $.40(.05)^{* * *}$ & $.37(.05)^{* * *}$ & $.32(.04)^{* * *}$ & $.08(.04)^{*}$ & $.15(.04)^{* * *}$ \\
\hline Financial Stressors & $.15(.05)^{* *}$ & $.15(.06)^{* *}$ & $.11(.04)^{* *}$ & $.07(.04)^{*}$ & $.08(.04)^{*}$ \\
\hline Illness-related Stressors & $-.06(.05)$ & $.10(.06)$ & $-.04(.04)$ & $.08(.04)^{*}$ & $-.02(.04)$ \\
\hline School-related Stressors & $.15(.04)^{* * *}$ & $.13(.05)^{* *}$ & $.08(.03)^{* *}$ & $.05(.03)$ & $.12(.03)^{* * *}$ \\
\hline$R^{2}$ & .16 & .17 & .18 & .06 & .08 \\
\hline
\end{tabular}

Note: $* p<.05 . * * p<.01 . * * * p<.001$

e.g., Cadigan, et al., 2019a, 2019b). The sample also scored above the scale midpoint on subjective perceptions of poor mental health. Similarly, the mean score on poor sleep quality was above the scale midpoint, indicating that young adults, on average, may be struggling with sleep health during this time.

Examining the mean scores of the five domains of COVID-19-related stressors, young adults reported the most concern regarding school-related stressors, social/relational stressors, and illness-related stressors, while reporting relatively lower financial and job-security stressors. As anticipated, each of these five stressor domains was generally correlated with indices of poorer mental health and well-being. Moreover, bivariate correlations indicated that stressors related to job insecurity, social/relational, and finances were positively correlated with weekly alcohol use and marijuana use frequency. Across each of the five domains, women reported greater endorsement of stressors, relative to men.

\section{Mental Health and Well-Being}

Regression models examining the associations between COVID-19-related stressors and indices of mental health and well-being are displayed in Table 3. Holding age, birth sex, and four-year/two-year student status constant across all models, we found that symptoms 
Table 4 Count regression models estimating associations between COVID-19 stressors and alcohol/marijuana use $(N=1181)$

\begin{tabular}{|c|c|c|c|}
\hline & $\begin{array}{c}\text { Typical Drinks per } \\
\text { Week (DDQ) }\end{array}$ & $\begin{array}{c}\text { Heavy Episodic } \\
\text { Drinking Occasions }\end{array}$ & $\begin{array}{c}\text { Marijuana Use } \\
\text { Frequency }\end{array}$ \\
\hline & Ratio $(95 \%$ CI $)$ & Ratio $(95 \% \mathrm{CI})$ & Ratio $(95 \% \mathrm{CI})$ \\
\hline \multicolumn{4}{|l|}{ Logistic Portion of the Models } \\
\hline Age & $0.68(0.62,0.73)^{* * *}$ & $0.83(0.75,0.92)^{* * *}$ & $0.86(0.80,0.93)^{* * *}$ \\
\hline Birth $\operatorname{Sex}(M=0, F=1)$ & $1.15(0.88,1.51)$ & $0.80(0.56,1.15)$ & $1.22(0.93,1.59)$ \\
\hline Student Status $(2$-year $=0,4$-year $=1)$ & $0.54(0.41,0.71)^{* * *}$ & $0.53(0.36,0.78)^{* *}$ & $1.03(0.79,1.35)$ \\
\hline Job Insecurity Stressors & $0.85(0.75,0.97)^{*}$ & $0.95(0.79,1.13)$ & $1.03(0.91,1.17)$ \\
\hline Social/Relational Stressors & $0.67(0.58,0.78)^{* * *}$ & $0.74(0.61,0.90)^{* *}$ & $0.85(0.74,0.98)^{*}$ \\
\hline Financial Stressors & $1.15(0.99,1.33)$ & $1.03(0.84,1.26)$ & $0.92(0.79,1.06)$ \\
\hline Illness-related Stressors & $1.18(1.01,1.37)^{*}$ & $1.04(0.84,1.28)$ & $1.00(0.86,1.17)$ \\
\hline School-related Stressors & $0.99(0.88,1.13)$ & $1.01(0.86,1.19)$ & $0.97(0.86,1.10)$ \\
\hline \multicolumn{4}{|l|}{ Count Portion of the Models } \\
\hline Age & $1.08(1.06,1.10)^{* * *}$ & $1.19(1.11,1.27)^{* * *}$ & $0.99(0.96,1.03)$ \\
\hline Birth $\operatorname{Sex}(M=0, F=1)$ & $0.80(0.75,0.86)^{* * *}$ & $0.74(0.59,0.92)^{* *}$ & $0.80(0.71,0.91)^{* * *}$ \\
\hline Student Status $(2$-year $=0,4$-year $=1)$ & $1.46(1.35,1.59)^{* * *}$ & $1.32(1.03,1.69)^{*}$ & $0.75(0.66,0.85)^{* * *}$ \\
\hline Job Insecurity Stressors & $1.03(1.00,1.07)$ & $1.12(1.01,1.26)^{*}$ & $1.02(0.96,1.08)$ \\
\hline Social/Relational Stressors & $1.16(1.12,1.21)^{* * *}$ & $1.29(1.14,1.47)^{* * *}$ & $1.04(0.97,1.11)$ \\
\hline Financial Stressors & $1.03(0.99,1.07)$ & $0.98(0.86,1.11)$ & $1.03(0.97,1.10)$ \\
\hline Illness-related Stressors & $0.95(0.91,1.00) *$ & $0.78(0.69,0.90)^{* * *}$ & $0.99(0.92,1.07)$ \\
\hline School-related Stressors & $0.95(0.92,0.99) * *$ & $0.86(0.79,0.94)^{* *}$ & $0.99(0.93,1.04)$ \\
\hline
\end{tabular}

Note: Ratios shown in the logistic portion of the models are zero-inflated odds ratios that estimate the likelihood of the outcome variable being a zero-value (i.e., values greater than one indicate that the covariate positively predicts a zero-value outcome). Ratios shown in the count portion of the models are Poisson incidence rate ratios, which represent proportional change in the outcome variable for each unit increase in the covariate (e.g., a ratio of $1.15=15 \%$ increase in the outcome variable for each unit change in the covariate). $* p<.05 . * * p<.01 . * * * p<.001$

of depression and anxiety were strongly associated with social/relational stressors, and moderately associated with financial and school-related stressors. These associations held for participants' subjective reports of overall mental health, with job insecurity stressors also being positively associated. Social/relational, financial, and school-related stressors were all associated with poorer sleep quality. These models generally supported hypothesizing that COVID-19-related stressors would be associated with poorer mental health and well-being, and revealed specific domains of stressors that are more closely related to these indices.

\section{Alcohol and Marijuana Use}

ZIP regression models estimating associations between COVID-19-related stressors and alcohol and marijuana use are displayed in Table 4. We again held the effects of age, birth sex, and four-year/two-year student status constant across these models. The logistic portion of these models revealed that those experiencing more job insecurity and social/relational stressors were less likely to abstain from weekly drinking (i.e., more likely to report engaging in any alcohol use). However, those who reported experiencing more illnessrelated stressors were more likely to abstain from weekly alcohol use. Social/relational stressors were also associated with greater likelihood of engaging in any heavy episodic drinking and greater likelihood of engaging in any marijuana use (i.e., non-zero response).

The count portion of the ZIP models estimated associations between COVID-19-related stressors and the amount of each substance use behavior. Job insecurity stressors were positively associated with more heavy episodic drinking occasions. Similarly, social/ 
relational stressors were positively associated with more drinks per week and more heavy episodic drinking occasions. Counter to our hypotheses, illness and school-related stressors were inversely associated with typical drinks per week and heavy episodic drinking occasions. Financial stressors were not significantly associated with alcohol or marijuana use and, counter to our expectations, marijuana use frequency was not significantly associated with any of the five COVID-19-related stressors, when estimated in these count regression models.

\section{Discussion}

In this study, we first identified distinct domains of COVID-19-related stressors for college students: job insecurity, social/relational, financial, illness-related, and school-related. We then examined associations between these stressors and indices of mental health and well-being, including substance use behaviors. Each of the five stressor domains was significantly associated with at least one indicator of mental health and well-being, with the overall pattern of results indicating that COVID-19-related stressors are related to poorer mental health and well-being. Social/relational stressors emerged as a primary concern as this domain was significantly associated with all five indices of mental health and wellbeing and, relative to other stressors, was most strongly associated with symptoms of depression and anxiety, poor mental health, and poor sleep quality. Social/relational stressors were also most strongly associated with greater weekly drinking and heavy episodic drinking, relative to other stressors. However, illness-related stressors and school-related stressors were associated with lower alcohol use, highlighting the value in disentangling the various domains of stressors that young adults may be experiencing during the COVID-19 pandemic. Interestingly, COVID-19-related stressors were not associated with marijuana use. Significant associations with each specific stressor domain are described in the following paragraphs.

The innate drive to feel connected with others is a fundamental human need (Baumeister \& Leary, 1995), and social relationships are of great importance during young adulthood (Arnett, 2015). Campus closures and social distancing measures broadly, have placed strain on young adults' social lives as seen in spikes in loneliness (Lee et al., 2020) and poorer mental health/well-being among those who felt more disconnected with and unsupported by their peer groups during the pandemic (Graupensperger et al., 2020). The current findings add to this growing concern: Social/relational stressors were strongly associated with depression and anxiety symptoms and subjective reports of poor mental health. Moreover, social/relational stressors were associated with poor subjective physical health and sleep quality, highlighting potential psychosomatic responses to this COVID-19-related stressor. Social/relational stressors were also associated with alcohol and marijuana use such that greater stress increased the likelihood of any alcohol use, any heavy episodic drinking, and any marijuana use (i.e., logistic portion of the ZIP model). Moreover, these models indicated that a oneunit increase in social/relational stressors was associated with a $16 \%$ increase in weekly drinking and a $29 \%$ increase in heavy episodic drinking. This finding aligns with recent findings that young adults' depression coping motives have increased during the pandemic (Graupensperger, Fleming, et al., 2021), and COVID-19-related psychological distress is associated with increased alcohol use (Rodriguez et al., 2020). These 
associations aid our understanding of how the social disconnectedness during the pandemic can have secondary negative implications for the health and well-being of young adults.

Relative to other age groups, young adults have faced greater job-loss and unemployment rates during the COVID-19 pandemic (Kochnar, 2020). The current findings show that job insecurity stressors (e.g., being laid-off or having hours reduced) was not significantly associated with symptoms of depression or anxiety, but was associated with poorer perceptions of global mental health, poorer perceptions of subjective physical health, and greater engagement in heavy episodic drinking. These results align with findings from a study in Israel, which found that COVID-19-related unemployment was associated with greater psychological distress among young adults (Achdut \& Refaeli, 2020). The finding that job insecurity stressors may have negative impacts on young adults' well-being is particularly troubling given that college students are more likely to be employed part-time and in industries more heavily impacted by the pandemic (e.g., service industry).

Job insecurity stressors were highly correlated with financial stressors; however, unlike job insecurity, financial stressors were significantly associated with all five indices of mental health and well-being. Food and housing insecurities are central components of financial stress, as currently operationalized, and prior studies have shown that these insecurities are associated with mental health concerns and sleep quality (Liu et al., 2014). Thus, the current findings extend previous research by focusing on financial stressors that are specific to the COVID-19 pandemic, and highlight the importance of policies that can assist young adults financially during these distressing times (e.g., rent relief, stimulus plans).

Counter to expectations based on findings from a study of Chinese college students ( $\mathrm{Li}$ et al., 2020), illness-related stressors were not significantly associated with indices of mental health, but were associated with subjective perceptions of poor physical health. Illnessrelated stressors were also associated with alcohol use, but not in the anticipated direction; those with greater illness-related stressors were more likely to abstain from weekly alcohol use and, after accounting for the excess zero responses, illness-related stressors were associated with fewer weekly drinks and fewer heavy episodic drinking occasions. Those college students who were more concerned with the disease itself, are plausibly more healthconscious individuals and perhaps more likely to avoid the social environments in which alcohol use is most common.

With colleges primarily moving to online formats, school-related stressors have been a primary concern for students. The current findings revealed positive associations between school-related stressors and depression, anxiety, poor subjective mental health, and poor sleep quality. These associations with students' mental health and well-being are deeply concerning considering the majority of US college students are likely to face continued disruptions to the higher education experience as the pandemic continues. However, school-related stressors were associated with less weekly drinking and fewer heavy episodic drinking occasions. While somewhat counter to expectations, this finding is intuitive as students who are more concerned about their schoolwork may engage in less alcohol use. A major implication of the current study is the identification of five unique domains of COVID-19-related stressors for college students, and that these domains demonstrated differential associations with indices of mental health/well-being and substance use. Although additional psychometric studies are needed, this COVID-19-related stressors scale may have vast utility for researchers studying the effects of the pandemic on college students.

Finally, an interesting finding that warrants further exploration is that COVID-19-related stressors were not associated with marijuana use frequency and social/relational stressors were only associated with increased likelihood of any marijuana use. It may be that young 
adult college students are not using marijuana to cope, but are instead choosing to drink when faced with COVID-19-related stressors, especially the social and job stressors. Moreover, an important consideration of these data/analyses is that they only detect effects that are averaged across the full sample. Indeed, some students may have increased marijuana use as a method of coping with stressors, while others may have decreased their marijuana use in response to increased stressors. Thus, person-level analyses that can disentangle individuals' responses to stressors from the sample's average effects may be prudent.

\section{Clinical Implications}

As young adulthood is characterized, in part, by changes to social roles and relationships (Schulenberg et al., 2002), stress related to social/relationships may be especially salient for this population. Young adults who are especially concerned about feeling isolated or alone and experience stressors pertaining to their social life or with those they live with, are a critically important group to identify and provide brief evidence-based strategies for improving mood and coping. Young adults may benefit from targeted COVID-focused prevention/intervention program focused on improving well-being by increasing social support, enhancing social connectedness, increasing coping skills for managing stress, and promoting low-risk substance use (Cadigan, under review). Concrete suggestions on ways to engage in socially distant social interactions, virtual interactions, and substancefree social activities could also be provided (Einberger et al., 2021). It is also concerning that many of the outcomes assessed in the present study are interrelated and problems in one domain (e.g., depressive symptoms) may likely have bidirectional influences on other domains (e.g., alcohol use). It will continue to be important to examine these downstream effects of mental health and substance use during the COVID-19 pandemic and incorporating clinical tools that may address both.

\section{Limitations and Future Directions}

Alongside the timeliness and clear implications of this research, results should be considered in the context of study sample, design, and timing. First, it is a strength to have included both four- and two-year college students in the current sample, especially given evidence that community college students face high levels of stress and may be at high risk of substance use (Cadigan et al., 2020, 2019a, b). The extent that these findings generalize to non-student young adults, in particular the subscale pertaining to school-related stressors is nevertheless unclear. Forthcoming studies could exclude the school-related stressors subscale and examine the effects of other COVID-19-related stressors on non-student young adults' mental health/well-being and substance use. Related to the measurement, the urgency of this research precluded us from conducting rigorous measurement testing on the COVID-19 stressors scale, and there were no validated scales available at this time. Another limitation to this study is that these findings captured the several months following the start of the pandemic, while circumstances have continued to evolve rapidly. This limitation is ubiquitous across COVID-19 studies, but it is important to consider whether college students will continue to experience similar levels of stress throughout the pandemic or if they will alternatively begin to adapt/adjust to the 'new normal'. Longitudinal research is thus needed to build upon these initial cross-sectional data. 
The relatively low response rate (i.e., 21.28\%) should be considered. Low response rate is not surprising given that this study recruited randomly selected students via e-mail (as opposed to more captive samples such as those collected in classes for credit, for example) and during a major global pandemic. Moreover, response rates are typically low when recruiting community college samples (Lee et al., 2021; Sax et al., 2008), but there is evidence that low response rates do not necessarily bias the results of survey-based studies (Fosnacht et al., 2017). Although many studies are reporting lower-than-usual response rates during the COVID-19 pandemic (e.g., Graupensperger et al., 2021a, 2021b), it is plausible that students facing more extreme distress would be less likely to participate.

\section{Conclusions}

Life disruptions associated with the COVID-19 pandemic may have myriad negative consequences to health and well-being that extend well beyond the SARS-CoV-2 virus. Although physical distancing measures that have impacted college-students are critical for reducing the spread of COVID-19, there is increasing evidence that these circumstances have been highly stressful for many college students. In the current study, we identified five distinct domains of COVID-19-related stressors and found that these stressors are associated with indices of mental health, well-being, and alcohol use. Importantly, associations varied across the five distinct stressors, with the evidence indicating that social/relational stressors may be having the most salient negative impact on college-students mental health and well-being. Although further evidence is needed, these findings provide valuable initial evidence to inform policy and translational strategies that can enhance college students' well-being during the current pandemic, and in preparation for future circumstances that may cause major disruptions to young adults' lives.

Supplementary Information The online version contains supplementary material available at https://doi. org/10.1007/s11469-021-00604-0.

Acknowledgements The authors would like to thank Dr. Devon Abdallah and Rose Lyles-Riebli for their help and coordination of data collection and Theresa Walter and Dr. Megan Patrick for their consultation on our measures.

Funding Data collection and manuscript preparation was supported by grants from the National Institute on Alcohol Abuse and Alcoholism (R01AA016979, R01AA027496-02S1, and T32AA007455) and the National Institute on Drug Abuse (R25DA050985). The content of this manuscript is solely the responsibility of the authors and does not necessarily represent the official views of the National Institutes of Health.

\section{Declarations}

Conflict of Interest The authors declare no competing interests.

\section{References}

ACHA. (2018). American College Health Association-National College Health Assessment II: Reference Group Executive Summary Fall 2018.

Achdut, N., \& Refaeli, T. (2020). Unemployment and psychological distress among young people during the COVID-19 pandemic: Psychological resources and risk factors. International Journal of En, 17, 7163. 
Arnett, J. J. (2015). Socialization in emerging adulthood: From the family to the wider world, from socialization to self-socialization. In J. E. Grusec \& P. D. Hastings (Eds.), Handbook of socialization: Theory and Research (pp. 85-105). The Guilford Press.

Bachman, J. G., Johnston, L. D., \& O’Malley, P. M. (2012). Monitoring the Future questionnaire responses from the nation's high school seniors. Ann Arbor: Institute for Social Research, The University of Michigan., at: http://www.monitoringthefuture.org/datavolumes.

Baumeister, R. F., \& Leary, M. R. (1995). The need to belong: Desire for interpersonal attachments as a fundamental human motivation. Psychological Bulletin, 117, 497-529. https://doi.org/10.1037/00332909.117.3.497

Bernaards, C., \& Jennrich, R. I. (2005). Gradient projection algorithms and software for arbitrary rotation criteria in factor analysis. Educational and Psychological Measurement, 65, 676-696. http://www.stat. ucla.edu/research/gpa.

Bovier, P. A., Chamot, E., \& Perneger, T. V. (2004). Perceived stress, internal resources, and social support as determinants of mental health among young adults. Quality of Life Research, 13, 161-170.

Bremner, J. (2020). U.S. alcohol sales increase 55 percent in one week amid coronavirus pandemic. Newsweek.

Cadigan, J. M., Duckworth, J. C., \& Lee, C. M. (2020). Physical and mental health issues facing community college students. Journal of American College Health, Ahead of Print. https://doi.org/10.1080/07448 481.2020.1776716

Cadigan, J. M., Dworkin, E. R., Ramirez, J. J., \& Lee, C. M. (2019a). Patterns of alcohol use and marijuana use among students at 2- and 4-year institutions. Journal of American College Health, 67, 383-390. https://doi.org/10.1080/07448481.2018.1484362

Cadigan, J. M., Lee, C. M., \& Larimer, M. E. (2019b). Young adult mental health: A prospective examination of service utilization, perceived unmet service needs, attitudes, and barriers to service use. Prevention Science, 20, 366-376.

Clay, J. M., \& Parker, M. O. (2020). Alcohol use and misuse during the COVID-19 pandemic: A potential public health crisis? The Lancet Public Health, 2667(20), 30088. https://doi.org/10.1016/S24682667(20)30088-8

Collins, R. L., Parks, G. A., \& Marlatt, G. A. (1985). Social determinants of alcohol consumption: The effects of social interaction and model status on the self-administration of alcohol. Journal of Consulting and Clinical Psychology, 53, 189-200.

Einberger, C., Graupensperger, S., \& Lee, C. M. (2021). Young adults' physical distancing behaviors during the initial months of the COVID-19 pandemic: Adherence to guidelines and associations with alcohol use behavior. Emerging Adulthood. https://doi.org/10.1177/21676968211004679

Fosnacht, K., Howe, E., \& Peck, L. K. (2017). How important are high response rates for college surveys? The Review of Higher Education, 40, 245-265.

Graupensperger, S., Benson, A. J., Kilmer, J. R., \& Evans, M. B. (2020). Social (un)distancing: Teammate interactions, athletic identity, and mental health of student-athletes during the COVID-19 pandemic. Journal of Adolescent Health, 67, 662-670.

Graupensperger, S., Fleming, C. B., Jaffe, A. E., Rhew, I. C., Patrick, M. E., \& Lee, C. M. (2021a). Changes in young adults' alcohol and marijuana use, norms, and motives from before to during the COVID-19 pandemic. Journal of Adolescent Health.

Graupensperger, S., Jaffe, A. E., Fleming, C. B., Kilmer, J. R., Lee, C. M., \& Larimer, M. E. (2021b). Changes in college student alcohol use during the COVID-19 pandemic: Are perceived drinking norms still relevant? Emerging Adulthood.

Holmes, E. A., O’Connor, R. C., Perry, V. H., Tracey, I., Wessely, S., Arseneault, L., Ballard, C., Christensen, H., Cohen Silver, R., Everall, I., Ford, T., John, A., Kabir, T., King, K., Madan, I., Michie, S., Przybylski, A. K., Shafran, R., Sweeney, A., ... Bullmore, E. (2020). Multidisciplinary research priorities for the COVID-19 pandemic: A call for action for mental health science. The Lancet Psychiatry, 0366, 1-14. https://doi.org/10.1016/S2215-0366(20)30168-1

Kessler, R. C., Berglund, P., Demler, O., Jin, R., Merikangas, K. R., \& Walters, E. E. (2005). Lifetime prevalence and age-of-onset distributions of DSM-IV disorders in the National Comorbidity Survey replication. Archives of General Psychiatry, 62, 593-602.

Khantzian, E. J. (1997). The self-medication hypothesis of substance use disorders: A reconsideration and recent applications. Harvard Review of Psychiatry, 4, 231-244.

Killgore, W. D. S., Cloonan, S. A., Taylor, E. C., \& Dailey, N. S. (2020). Loneliness: A signature mental health concern in the era of COVID-19. Psychiatry Research, 290, 113117. https://doi.org/10.1016/j. psychres.2020.113117

Kochnar, R. (2020). Unemployment rose higher in three months of COVID-19 than it did in two years of the Great Recession. Pew Research Center, June 11, 2020. 
Kroenke, K., Spitzer, R. L., Williams, J. B. W., \& Löwe, B. (2009). An ultra-brief screening scale for anxiety and depression: The PHQ-4. Psychosomatics, 50, 613-621. https://doi.org/10.1016/S00333182(09)70864-3

Lechner, W. V., Laurene, K. R., Patel, S., Anderson, M., Grega, C., \& Kenne, D. R. (2020). Changes in alcohol use as a function of psychological distress and social support following COVID-19 related university closings. Addictive Behaviors, 110, 106527. https://doi.org/10.1016/j.addbeh.2020.106527

Lee, C. M., Cadigan, J. M., \& Rhew, I. C. (2020). Increases in loneliness among young adults during the COVID-19 pandemic and association with increases in mental health problems. Journal of Adolescent Health, Ahead of Print.

Lee, Christine M., Cadigan, J. M., Kilmer, J. R., Cronce, J. M., Suffoletto, B., Walter, T., Fleming, C. B., \& Lewis, M. A. (2021). Brief Alcohol Screening and Intervention for Community College Students (BASICCS): Feasibility and preliminary efficacy of web-conferencing BASICCS and supporting automated text messages. Psychology of Addictive Behaviors, Ahead of Print. https://doi.org/10.1037/ $\mathrm{adb} 0000745$

Li, H. Y., Cao, H., \& Leung, D. Y. P. (2020). The psychological impacts of a COVID-19 outbreak on college students in China: A longitudinal study. International Journal of Environmental Research and Public Health, 17, 3933.

Liu, Y., Njai, R. S., Greenlund, K. J., Chapman, D. P., \& Croft, J. B. (2014). Relationships between housing and food insecurity, frequent mental distress, and insufficient sleep among adults in 12 US States. Preventing Chronic Disease, 11, 1-9.

Luchetti, M., Lee, J. H., Aschwanden, D., Sesker, A., Strickhouser, J. E., Terracciano, A., \& Sutin, A. R. (2020). The trajectory of loneliness in response to COVID-19. American Psychologist, Ahead of Print. doi: https://doi.org/10.1037/amp0000690.

McGinty, E. E., Presskreischer, R., Han, H., \& L., B. C. . (2020). Psychological distress and loneliness reported by US adults in 2018 and April 2020. JAMA. https://doi.org/10.1001/jama.2020.9740

Pfefferbaum, B., \& North, C. S. (2020). Mental Health and the Covid-19 Pandemic. New Englan, 383, 510512. https://doi.org/10.1056/NEJMp2013466

Rodriguez, L. M., Litt, D. M., \& Stewart, S. H. (2020). Drinking to cope with the pandemic: The unique associations of COVID-19-related perceived threat and psychological distress to drinking behaviors in American men and women. Addictive Behaviors, 110, 106532. https://doi.org/10.1016/j.addbeh.2020. 106532

Sax, L. J., Gilmartin, S. K., Lee, J. J., \& Hagedorn, L. S. (2008). Using web surveys to reach community college students: An analysis of response rates and response bias. Community College Journal of Research and Practice, 32, 712-729. https://doi.org/10.1080/10668920802000423

Schulenberg, J. E., Ph, D., Maggs, J. L., \& Ph, D. (2002). A developmental perspective on alcohol use and heavy drinking during adolescence and the transition to young adulthood. Journal of Studies on Alcohol and Drugs, Supplement, 54-70.

Serido, J., Almeida, D. M., \& Wethington, E. (2004). Chronic stressors and daily hassles: Unique and interactive relationships with psychological distress. Journal of Health and Social Behavior, 45, 17-33.

Tworoger, S. S., Davis, S., Vitiello, M. V., Lentz, M. J., \& Mctiernan, A. (2005). Factors associated with objective (actigraphic) and subjective sleep quality in young adult women. Journal of Psychosomatic Research, 59, 11-19. https://doi.org/10.1016/j.jpsychores.2005.03.008

Vuong, Q. H. (1989). Likelihood ratio tests for model selection and non-nested hypotheses. Econometrica, 57, 307-333.

Weitzman, E. R. (2004). Poor mental health, depression, and associations with alcohol consumption, harm, and abuse in a national sample of young adults in college. The Journal of Nervous and Mental Disease, 192, 269-277. https://doi.org/10.1097/01.nmd.0000120885.17362.94

Wu, L. T., Pilowsky, D. J., Schlenger, W. E., \& Hasin, D. (2007). Alcohol use disorders and the use of treatment services among college-age young adults. Psychiatric Services, 58, 192-200.

Publisher's Note Springer Nature remains neutral with regard to jurisdictional claims in published maps and institutional affiliations. 